\title{
El modelo de gestión en red para la atención integral de la primera infancia en Costa Rica: Una mirada desde sus actores
}

\author{
The Network Management Model for Comprehensive Early Childhood Care in Costa Rica: A \\ View From its Actors
}

O modelo de gestão em rede para a atenção integral à primeira infância na Costa Rica: Uma visão a partir de seus atores

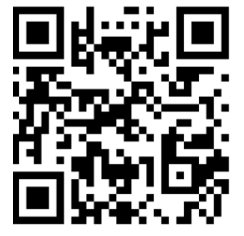

Dora Hernández-Vargas

Universidad Nacional

ROR:: https://ror.org/01t466c14

Heredia, Costa Rica

dorahv@gmail.com

https://orcid.org/0000-0003-2765-5042

Manuel Chacón-Ortiz

Universidad Nacional

ROR:.: https://ror.org/01t466c14

Heredia, Costa Rica

manuch@gmail.com

https://orcid.org/0000-0002-0758-3275

Recibido • Received • Recebido: 24 / 11 / 2020

Corregido • Revised • Revisado: 07 / 06 / 2021

Aceptado • Accepted • Aprovado: 05 / 07 / 2021

\begin{abstract}
Resumen: Este estudio analiza los resultados de una encuesta llevada a cabo a profesionales que coordinan redes de atención intersectorial en el marco del Sistema de Atención Integral e Intersectorial del Desarrollo de Niñas y Niños (SAIID) en Costa Rica. La encuesta tuvo como objetivo caracterizar la experiencia costarricense del modelo de gestión en red desde la perspectiva de sus actores. La metodología utilizada tiene un enfoque cuantitativo y reporta un estudio exploratorio de diseño no experimental. La información fue recabada mediante un cuestionario en línea aplicando el criterio de muestreo a conveniencia. El instrumento fue aplicado a 43 personas coordinadoras de redes regionales y locales participantes de la iniciativa del SAIID. Los resultados muestran que la experiencia de trabajo en red del SAIID se ha basado en un modelo intersectorial de gestión más flexible en relación con otros modelos tradicionales, centralistas y verticales. Las personas participantes reportaron que este modelo fortaleció el desarrollo de sus capacidades de liderazgo en todos los niveles. Dada la variedad de instancias estatales implicadas, los procesos de gestión conllevaron esfuerzos de planificación, así como evaluación permanente; apoyada por protocolos de atención y distintas herramientas para la valoración y el seguimiento intersectorial del desarrollo de la niñez. Se concluye que un modelo de gestión que apuesta por la participación interdisciplinaria y la formación continua de quienes son parte del sistema resulta un modelo sostenible que da respuesta a los llamados de la comunidad nacional e internacional por el cuidado y la educación de la infancia.
\end{abstract}

Palabras clave: Sistemas de atención a la primera infancia; articulación intersectorial; modelos de gestión en red; atención integral de la niñez; trabajo en red. 
http://doi.org/10.15359/ree.25-3.12

http://www.una.ac.cr/educare

educare@una.ac.cr

\begin{abstract}
This study analyzes the results of a survey carried out among professional coordinators of intersectoral care networks within the framework of the Sistema de Atención Integral e Intersectorial del Desarrollo de Niñas y Niños (SAIID) in Costa Rica. The objective of the survey was to characterize the Costa Rican experience of the network management model from the perspective of its actors. The methodology used has a quantitative approach and reports an exploratory study of non-experimental design. The information was collected through an online questionnaire applying the convenience sampling criterion. The instrument was applied to 43 coordinators of regional and local networks participating in the SAIID initiative. The results show that SAlID's networking experience has been based on a more flexible intersectoral management model in relation to other traditional, centralist, and vertical models. Participants reported that this model strengthened the development of their leadership skills at all levels. Given the variety of state entities involved, the management processes involved planning efforts and permanent evaluation, supported by care protocols and different tools for the assessment and intersectoral monitoring of childhood development. It is concluded that a management model that is committed to interdisciplinary participation and continuous training of those who are part of the system is a sustainable model that responds to the calls of the national and international community for the care and education of children.
\end{abstract}

Keywords: Early childhood care systems; intersectoral articulation; network management models; comprehensive childhood care; networking.

Resumo: Este estudo analisa os resultados de uma pesquisa realizada com coordenadores profissionais de redes intersetoriais de atenção no âmbito do Sistema de Atenção Integral e Intersetorial do desenvolvimento de crianças (SAIID) da Costa Rica. O objetivo da pesquisa foi caracterizar a experiência costarriquenha do modelo de gestão de redes a partir da perspectiva de seus atores. A metodologia utilizada tem abordagem quantitativa e relata um estudo exploratório de delineamento não experimental. As informações foram coletadas por meio de questionário online, aplicando-se o critério de amostragem por conveniência. $\mathrm{O}$ instrumento foi aplicado a 43 coordenadores de redes regionais e locais participantes da iniciativa SAIID. Os resultados mostram que a experiência de networking do SAIID tem se baseado em um modelo de gestão intersetorial mais flexível em relação a outros modelos tradicionais, centralistas e verticais. Os participantes relataram que esse modelo fortaleceu o desenvolvimento de suas habilidades de liderança em todos os níveis. Dada a variedade de entidades estaduais envolvidas, os processos de gestão uniram esforços de planejamento, bem como avaliação permanente; respaldada por protocolos de atenção e diferentes ferramentas de avaliação e acompanhamento intersetorial do desenvolvimento infantil. Conclui-se que um modelo de gestão comprometido com a participação interdisciplinar e a formação continuada de quem faz parte do sistema é um modelo sustentável que responde aos apelos da comunidade nacional e internacional para o cuidado e a educação das crianças.

Palavras-chave: Sistemas de atenção à primeira infância; articulação intersetorial; modelos de gestão de rede; atenção integral à infância, rede. 
http://doi.org/10.15359/ree.25-3.12

\section{Introducción}

La Convención de los Derechos del Niño señala la importancia de que los países promuevan el desarrollo de la niñez de forma coordinada y de manera multisectorial (Fondo de las Naciones Unidas para la Infancia [UNICEF] y Sistema Nacional para el Desarrollo Integral de la Familia [DIF], 2001). De acuerdo con Zero to Three (2020), la primera infancia necesita acceso a servicios de atención que incluyan educación, salud y servicios de apoyo familiar asequibles y de calidad. Los programas y servicios que abordan estas áreas son fundamentales; sin embargo, su fortaleza está en la infraestructura que los respalda. Para ser eficaces, los programas deben organizarse dentro de sistemas cohesivos que coordinen y articulen una amplia gama de servicios. Para apoyar el desarrollo integral y saludable de la niñez, los Estados y las comunidades deben proporcionar sistemas intersectoriales, coordinados y bien financiados de servicios de alta calidad que fomenten el éxito en la escuela y en la vida. La conformación de redes intersectoriales para la atención de los determinantes de la salud y el desarrollo infantil ha sido una política en países desarrollados como Canadá, Australia y Holanda, pero es poco sistematizada y estudiada en Latinoamérica. La bibliografía académica apunta a un vacío sobre el cómo se estructuran estas redes intersectoriales, no menos importante, cómo estas funcionan de acuerdo con cada país y cada contexto (Anaf et al., 2014).

\section{La educación en concierto con un enfoque de atención integral y sistémico}

Hoy día, los distintos grupos profesionales reconocen la importancia de la educación y atención en los primeros años como una etapa fundamental en la creación de las bases madurativas y neurológicas del desarrollo y el aprendizaje. Términos como atención integral, desarrollo, o aprendizaje temprano son utilizados por personas educadoras, tanto como por profesionales de la psicología, de la salud, de la sociología y el trabajo social, quienes también participan del cuidado y atención de la primera infancia. La educación, desde esta nueva comprensión de la complejidad de la atención integral de la niñez, se enfrenta a nuevos retos y oportunidades. En el ámbito educativo existen dos perspectivas: una que considera la educación formal como actividad primariamente académica, escolarizante y garante de conocimientos y, por tanto, más centrada en el currículo y las instituciones; y otra que aboga por atender las necesidades integrales de los niños y las niñas, más centrada en su desarrollo y bienestar integral. Desde esta segunda perspectiva, y con un enfoque que considera el abordaje sistémico como la mejor forma de dar respuesta a las necesidades de la primera infancia, han surgido iniciativas de atención intersectorial y en red.

Un sistema de atención integral para la niñez debe estar basado en todas las dimensiones del ser humano: lo físico y lo emocional, considerando incluso la salud mental (Kagan y Kauerz, 2012). Es difícil encontrar un balance entre los sectores y actores institucionales que lo permitan, a su vez, en el marco de colaboración, autonomía e independencia de las partes. Aspectos tales como la coordinación e incidencia en políticas públicas han sido vacíos de gestión identificados (Bilodeau et al., 2018). Las colaboraciones intersectoriales exitosas tienen definidas con claridad sus metas, donde el contexto determina, en gran medida, las posibilidades de esta colaboración. 
http://doi.org/10.15359/ree.25-3.12

http://www.una.ac.cr/educare

educare@una.ac.cr

No obstante, se reconoce que el currículo educativo ya se encuentra bastante saturado de temas, y las acciones intersectoriales pueden representar una carga docente adicional (Tooher et al., 2016). En este contexto, se ha evidenciado que los estilos de liderazgo son un elemento clave que pueden definir el éxito o fracaso de esta colaboración (Pucher et al., 2015). Estas alianzas deben darse en el marco de una buena comunicación e información, buenas relaciones interpersonales, mediante la participación de informantes clave, y una mejor administración del tiempo (Burgess et al., 2016).

\section{Sistemas de atención intersectorial}

La integración de servicios para la niñez tiende a conducir a políticas más coherentes, mayor profesionalismo, mayores requisitos de calificación y mejores salarios (Kaga et al., 2010). Siendo así, es necesario que el sistema que atiende a la niñez genere competencias profesionales y colaborativas entre quienes lo conforman (Urban et al., 2012). Un sistema de atención integral a la niñez no puede ser la suma de las distintas instancias o instituciones que velan por la salud, educación o protección de los niños y niñas; debe constituirse en un organismo con fines comunes cuyos sujetos participantes trabajen desde la articulación intersectorial. De acuerdo con CunillGrau (2014), intersectorialidad significa "la relación entre diversos sectores gubernamentales" (p. 7); es decir, distintas instancias, públicas (o bien privadas o mixtas) relacionadas con la atención de la niñez, desde distintas disciplinas, que abordan temas comunes asociados con la atención integral mediante un enfoque holístico y sistémico. La atención integral procura que cada uno de estos sectores actúe en correspondencia con sus funciones propias, como lo son la prevención de enfermedades, educación oportuna, protección legal, entre otras. Sin abandonar estas funciones, lo anterior significa que los sectores "'se pongan de acuerdo' para actuar 'conjuntamente' a fin de lograr un cambio social respecto de la situación inicial" (Cunill-Grau, 2014, p. 8).

Algunos estudios reportan iniciativas de articulación intersectorial en diferentes países. Tales estudios muestran que, pese a sus variados modelos de articulación, la filosofía de trabajar de manera integrada y colaborativa resulta esencial para la prestación de servicios de alta calidad (Rentzou et al., 2019). Por ejemplo, Bélgica, Italia, Portugal y Eslovenia participaron en el proyecto INTESYS durante el 2015 al 2019, en el que la International Step by Step Association (ISSA) estimuló la construcción conjunta de un camino para la articulación intersectorial. Los resultados del proyecto revelaron que no existe una única vía para consolidar una propuesta de atención intersectorial. Tal modelo "puede ser ascendente (prestación en primera línea, comunidad, padres) o descendente (estrategias, políticas y gobernanza entre las distintas agencias)" (Rentzou et al., 2019, p. 70). Sin embargo, esta experiencia identificó que todo modelo que apueste por la integración debe basarse en tres componentes:"1. una visión e ideas compartidas entre las distintas partes implicadas; 2 . una serie de factores clave que fomenten la integración; y 3. prácticas de calidad" (Rentzou et al., 2019, p. 70).

Otro ejemplo de modelo de sistema para la atención intersectorial de la primera infancia en Latinoamérica es el caso de Chile. El Sistema Intersectorial de Protección Social de Chile, integrado 
http://doi.org/10.15359/ree.25-3.12

por dos subsistemas (Chile Solidario y Chile Cre-ce Contigo), integra redes como dispositivos a nivel comunal, coordinadas por las municipalidades. En este modelo, la articulación nacional se produce verticalmente, mientras que la articulación local permite un amplio margen de acción y decisión. Se concluye que en este caso, "en vez de un modelo descentralizado opera una dinámica de arriba-abajo, en la que se busca una lógica de integración centralmente dirigida" (Cunill-Grau, 2014, p. 29). El Sistema de Atención Integral Intersectorial del Desarrollo de Niñas y Niños en Costa Rica representa un esfuerzo por articular distintas instancias en la atención integral de la niñez.

\section{Sistema de Atención Integral Intersectorial del Desarrollo de Niñas y Niños en Costa Rica}

En el año 2005 se plantea una iniciativa de atención intersectorial, conformada por el Ministerio de Educación Pública (MEP), la Caja Costarricense del Seguro Social (CCSS), el Ministerio de Salud (MS) y la Dirección Nacional de los Centros de Educación y Nutrición y de Centros Infantiles de Atención Integral (CEN/CINAI). En 2010 se confiere el aval político a dicha iniciativa, formalizando así el Sistema de Atención Integral e Intersectorial del Desarrollo de Niñas y Niños (SAIID), al cual se unieron la Universidad de Costa Rica (UCR), la Universidad Nacional (UNA) y la Universidad Estatal a Distancia (UNED). Para el año 2015, este sistema es ratificado por las autoridades de educación y salud del país mediante oficio firmado por los jerarcas ministeriales (Despacho Ministerio de Educación Pública N. ${ }^{\circ}$ DM-1379-08-15; Despacho Ministro de Salud DM 7439-2015; Presidencia Ejecutiva CCSS P.E.47.303-15). En 2017 se firma un convenio de cooperación intersectorial entre el MEP, la CCSS, el MS y el Consejo Nacional de Rectores (CONARE), que ratificó las bases de esta cooperación recíproca en los escenarios de salud y educación.

EI SAIID propone una organización en red por niveles. A nivel local, las redes asumen tareas específicas, dentro de las que se destacan, en particular: la detección temprana de alteraciones de desarrollo, el manejo adecuado de cada caso y su derivación oportuna según criterios de referencia, el seguimiento de casos aprovechando al máximo los recursos disponibles, y el uso de herramientas o instrumentos comunes para la detección y derivación. A nivel regional, cada red es responsable de la conducción del proceso "considerando tanto las tareas tácticas regionales como las operativas locales" (Cerdas González, 2016, p. 45). Este sistema contempla el apoyo y acompañamiento a las familias, con énfasis en la niñez expuesta a mayores riesgos biopsicosociales (Cerdas González, 2016). A nivel nacional, un equipo con representación de todas las instituciones participantes lidera el proceso.

\section{Modelo de gestión del trabajo en red en Costa Rica}

El modelo de gestión del trabajo en red que supone el SAIID se estructura mediante la configuración de equipos intersectoriales e interdisciplinarios llamados RISA (Redes Integradas de Servicios de Atención). De esta forma, el SAIID permite que las redes de servicios de salud y educación funcionen de manera articulada; es decir, que las instituciones que atienden de manera directa a la niñez (CCSS-MEP-CEN/CINAI) se anuden en un accionar sistémico. De acuerdo con Cerdas González (2016, citando al SAIID, 2012), la estrategia de acción de estas RISA 
http://doi.org/10.15359/ree.25-3.12

http://www.una.ac.cr/educare

educare@una.ac.cr

"comprende la detección, prevención, abordaje, rehabilitación y seguimiento de las alteraciones o trastornos que se presentan en el desarrollo, salud y aprendizaje de niñas y niños" (p. 27).

La transformación del modelo de gestión conlleva un cambio en las prácticas y relaciones de los colectivos (Secretaría de Educación Pública [SEP], 2010). Esto implica una planificación estratégica dentro de las instituciones, y requiere la evaluación de las actividades regularmente ejecutadas, y genera procesos de realimentación, priorización y mejora. En el caso de Costa Rica ha necesitado "la elaboración de protocolos intersectoriales con criterios de calidad que permitan planificar, dirigir, ejecutar y evaluar los procesos continuamente" (Caja Costarricense de Seguro Social [CCSS] et al., 2012, p. 57).

Provan y Kenis (2008, citados por Cunill-Grau, 2014) admiten que existe mucha información sobre las virtudes de las redes, pero se ha escrito poco acerca de cómo su funcionamiento impacta en su efectividad. Con el fin de caracterizar la experiencia costarricense del modelo de gestión en red, este estudio explora las percepciones de equipos coordinadores regionales y locales de las redes (RISA) sobre las características que tuvo el modelo de gestión en red en el marco del SAIID durante el 2015. Con ello se pretende develar los elementos de gestión que han influido en su labor y la de sus equipos.

\section{Metodología}

De acuerdo con Alvira Martín (2011), la encuesta es la metodología de investigación más utilizada en ciencias sociales. Los diseños de investigación tipo encuesta son administrados a una muestra o al total de la población con el fin de describir las actitudes, opiniones, conductas o características de la población (Creswell, 2008). Este estudio en específico es de enfoque cuantitativo, de corte no experimental y de alcance exploratorio.

Diseño. En el diseño de encuesta de corte no experimental no seconstruye ninguna situación, sino que se observan situaciones ya existentes, las cuales no son provocadas intencionalmente por quienes investigan (Hernández Sampieri et al., 2014). No se explora la causalidad y no existen variables dependientes e independientes (Rovai et al., 2013). Su alcance es exploratorio, y podrá dar paso a estudios de mayor profundidad en torno a la experiencia investigada.

Técnica e instrumento de recolección de la información. La técnica empleada para la recolección de información fue una encuesta. Para este fin se llevó a cabo un muestreo a conveniencia (Creswell, 2008). En el muestreo a conveniencia el ente investigador selecciona al grupo participante porque tiene las características de constituirse en un grupo particular con información clave, aunque la muestra no sea representativa.

El instrumento para recabar los datos fue un cuestionario en línea. Se empleó el software LimeSurvey alojado en la plataforma del sitio web temporal llamado redsaiid.com. Las razones 
http://doi.org/10.15359/ree.25-3.12

http://www.una.ac.cr/educare educare@una.ac.cr

para emplear esta plataforma fueron varias. La primera, las personas que integran esta red se encuentran en distintas partes del país. Esta estrategia además aseguraba poder solventar las barreras geográficas y de recolección de información, por lo que la visita a estas comunidades o el envío físico del instrumento no se consideró la forma óptima de recolectar la información. Así mismo, los cuestionarios en línea permiten recolectar información de forma más fidedigna y procesar los datos con mayor facilidad (Hewson et al., 2016). El enlace al cuestionario fue enviado a las personas vía correo electrónico. Posterior a la aplicación del instrumento, la información fue tabulada para realizar el respectivo análisis de los datos y establecer las conclusiones del estudio. Para procesar con mayor precisión los datos se empleó el software SPSS.

El cuestionario en línea estuvo compuesto de preguntas abiertas y cerradas, diseñado a partir de las siguientes secciones, ordenadas en categorías:

1. Sección A: Datos generales de los miembros del equipo coordinador RISA

2. Sección B: Uso de documentos de normalización técnica

a. Herramientas de atención, protocolos, guías o lineamientos creados para uso intersectorial compartidas entre miembros de la RISA.

b. Base de datos que puede acceder el público usuario para conocer el seguimiento intersectorial que se da en cada caso (acción para superar la barrera acceso a la información por parte de los usuarios).

3. Sección C: Gestión del equipo coordinador

a. Mecanismos, actividades, estrategias implementadas por miembros de la red para mejorar la atención de las personas participantes.

b. Estrategias implementadas por el equipo coordinador para contrarrestar procedimientos o lineamientos que evidencian una organización institucional lineal, centralista y desarticulada que afectan la gestión en red.

c. Estrategias empleadas por las coordinaciones para el manejo de convocatorias a reuniones o capacitaciones y las estrategias para garantizar manejo de información oportuna por parte de jefaturas locales y regionales.

d. Herramientas de gestión, planificación y autoevaluación compartidas aplicadas por el equipo coordinador para articular una estrategia continua, periódica, integral.

4. Sección D: FODA en la gestión de casos intersectorial

a. Fortalezas y debilidades del proceso de acuerdo con las coordinaciones (condiciones internas).

b. Oportunidades y amenazas del proceso de acuerdo con las coordinaciones (condiciones externas al proceso). 
http://doi.org/10.15359/ree.25-3.12

http://www.una.ac.cr/educare

educare@una.ac.cr

Muestra. El criterio que se utilizó para la selección de personas participantes fue un criterio de muestra por conveniencia o muestreo estratégico (Cea D'Ancona, 1998) en el que las personas participantes fueron seleccionadas de manera intencional, debido a la relación que guardan con el objeto de estudio. El criterio para la selección de la muestra consistió en ubicar participantes de las diferentes disciplinas involucradas en las redes y de las diferentes redes presentes en el país, de manera que la muestra tuviera algún nivel de representatividad. Siendo que la cantidad total aproximada de miembros de las redes en todo el país para ese entonces era de 148 coordinadores, la muestra representa un 63\% de la población total.

Participantes. Para la recolección de información se trabajó con un grupo de informantes clave que conformaban las RISA. El cuestionario fue enviado a la coordinación de 19 RISA que funcionaba en el año 2015 en el momento de colectar la información. Un total de 43 sujetos coordinadores de redes regionales y locales accedieron a completar el instrumento. En la Tabla 1 se describen las características de las personas participantes. Cabe destacar que, a excepción de la Figura 1, en todas las tablas y figuras la cantidad de respuestas corresponde a la cantidad de menciones que hacen las personas participantes de las variables identificadas.

Tabla 1: Características de las personas participantes

\begin{tabular}{llrc}
\hline & & N & Porcentaje \\
\hline \multirow{4}{*}{ Sexo } & Mujer & 38 & 88.4 \\
& Hombre & 5 & 11.6 \\
\hline \multirow{4}{*}{ Profesión } & Preescolar & 24 & 55.8 \\
& Terapista lenguaje & 2 & 4.7 \\
& Médico & 5 & 11.6 \\
& Odontología & 4 & 9.3 \\
& Enfermería & 5 & 11.6 \\
& Nutrición & 1 & 2.3 \\
& Ed. especial & 1 & 2.3 \\
Grado Académico & 1 & 2.3 \\
& Psicología & 22 & 51.2 \\
& Licenciatura & 17 & 39.5 \\
& Maestría & 4 & 9.3 \\
\hline \multirow{4}{*}{ Institución } & Doctorado & 19 & 44.2 \\
& MEP-Centro Educativo & 8 & 18.6 \\
& CEN-CINAl & 14 & 32.6 \\
& CCSS & 2 & 4.7 \\
\hline
\end{tabular}

Nota: Elaboración propia con base en los resultados. 
http://doi.org/10.15359/ree.25-3.12

Consentimiento informado. La participación en el estudio fue voluntaria. La solicitud para contestar el cuestionario y el consentimiento fue expuesta antes de contestar el instrumento en línea y vía correo electrónico. Todas las personas participantes eran mayores de edad al momento de brindar información para el presente estudio y se les aseguró la confidencialidad en el uso de la información.

Alcances el estudio. Dada las decisiones metodológicas adoptadas, la población, diseño, muestra y tipo de análisis, el estudio no es generalizable. No se aplican en este estudio datos de validez y fiabilidad del instrumento, debido que se exponen de forma exploratoria, y no se ejecuta algún análisis que requiera demostrar estos criterios estadísticos. Este estudio se desarrolló en el año 2015, en un momento en el que las redes intersectoriales estaban en proceso de consolidación, y como respuesta al vacío de información sobre la forma en que las personas participantes percibían este modelo de gestión en red.

\section{Resultados}

Con el fin de caracterizar la experiencia costarricense del modelo de gestión en red, este estudio valora el manejo de documentos técnicos para el trabajo en red, seguido de la gestión de los equipos intersectoriales y, finalmente, la sistematización de las fortalezas, debilidades, oportunidades y amenazas percibidas por las coordinaciones de las RISA que participaron en la recolección de la información.

En relación con los documentos de estandarización y normalización técnica, las personas participantes consideraron que el documento mayormente empleado por las personas coordinadoras es la Boleta de Referencia y Contrarreferencia CCSS-MEP-MS empleada en un 58\% de las ocasiones, seguido del Expediente Educativo del MEP en un 51\% y el documento de Lineamientos para la Valoración del Desarrollo que aparecen en Registros Docentes (MEP) (Figura 1).

En relación con los mecanismos, actividades y estrategias implementados por miembros de las redes, las personas participantes manifestaron que los procesos de autogestión, así como las capacitaciones, son los mecanismos o estrategias más utilizados para mejorar la atención de los equipos (Figura 2).

Por su parte, la articulación y coordinación, así como la autogestión, fueron identificadas por quienes participaron como las estrategias más utilizadas para contrarrestar la organización institucional lineal, centralista y desarticulada que afecta la gestión en red (Figura 3). 
http://doi.org/10.15359/ree.25-3.12

http://www.una.ac.cr/educare

educare@una.ac.cr

Figura 1: Documentos de normalización técnica implementados por las coordinaciones

Manual o protocolo de manejo de situaciones de violencia (MEP)

Guía de Atención Integral a las Personas (componente niño-CCSS, versión 2000)

Guía para La Atención Integral de Niñas, Niños y Adolescentes Agredidos (CCSS, versión 2012)

Expediente pediátrico para uso del Primer Nivel de Atención (CCSS)

Carné rotafolio y video de Desarrollo Integral de la niña y el niño, divulgativo (CCSS-MEP-MS)

Documento Atención integral del desarrollo de niñas y niños en Costa Rica: fortalecimiento intersectorial (SAIID, versión, 2012)

Expediente Salud del Niño(a) CEN-CINAI

Lineamientos para la valoración del desarrollo que aparecen en Registros Docentes (MEP)

Expediente Educativo (MEP)

Boleta de referencia y contrarreferencia CCSS-MEP-MS (versión piloto)

16.3 25.6 20.9 37.2

\begin{tabular}{l|l|l|l}
18.6 & 39.5 & 20.9 & 20.9
\end{tabular}

\begin{tabular}{l|l|l|l}
20.9 & 30.2 & 18.6 & 30.2
\end{tabular}

\section{3} 23.3

18.6

34.9

\begin{tabular}{l|l|l|l}
\hline 23.3 & 32.6 & 20.9 & 23.3
\end{tabular}

\section{6}

37.2

18.6

11.6

\section{)}

\begin{tabular}{|l|l|l|l|}
\hline 46.5 & 11.6 & 16.3 & 25.6 \\
\hline
\end{tabular}

\begin{tabular}{|l|l|l|l|}
\hline 48.8 & 14 & 16.3 & 20.9 \\
\hline & & & \\
\hline 51.2 & 14 & 14 & 20.9 \\
\hline
\end{tabular}

\begin{tabular}{|l|l|l|l|l|}
\hline 58.1 & & 11.6 & 16.3 & 14 \\
\hline 0 & 25 & 50 & 75 & 100
\end{tabular}

Usualmente. En un 90\% de las ocasiones en que se han presentado un caso relacionado.

De vez en cuando. Entre un 50 y un 70\% de las veces en que se ha presentado un caso.

Rara Vez. Menos de un 30\% de las veces en que se ha presentado un caso.

Nunca

Nota: Elaboración propia con base en el instrumento.

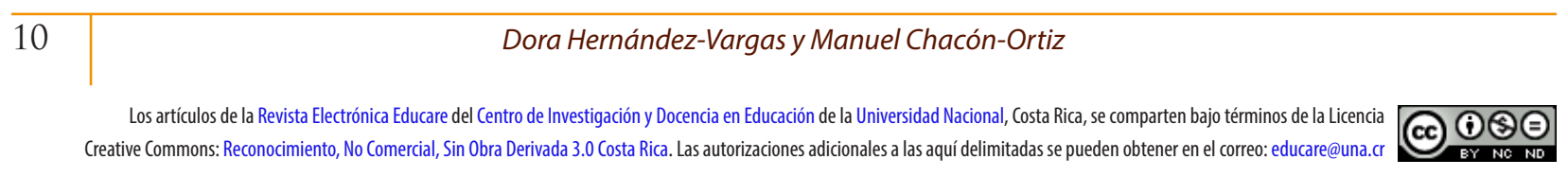


http://doi.org/10.15359/ree.25-3.12

http://www.una.ac.cr/educare educare@una.ac.cr

Figura 2: Mecanismos, actividades, estrategias implementadas por los miembros de la red para mejorar la atención de las personas participantes*

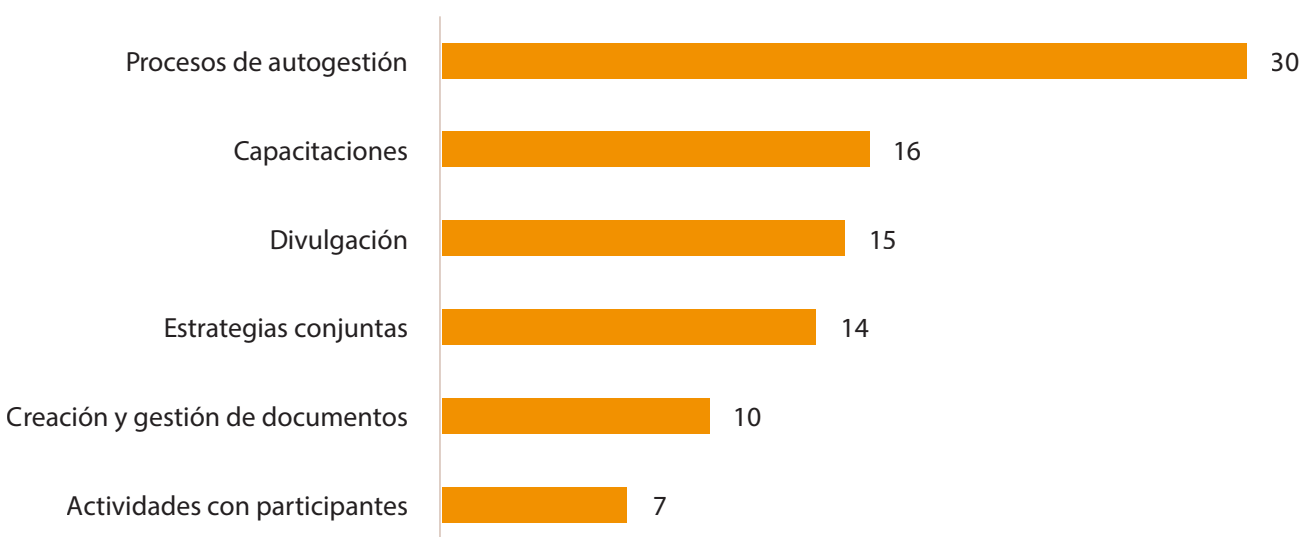

Nota: elaboración propia con base en el instrumento (*Representado en números reales)

Figura 3: Estrategias implementadas para contrarrestar procedimientos o lineamientos de una organización institucional lineal, centralista y desarticulada que afectan la gestión en red*

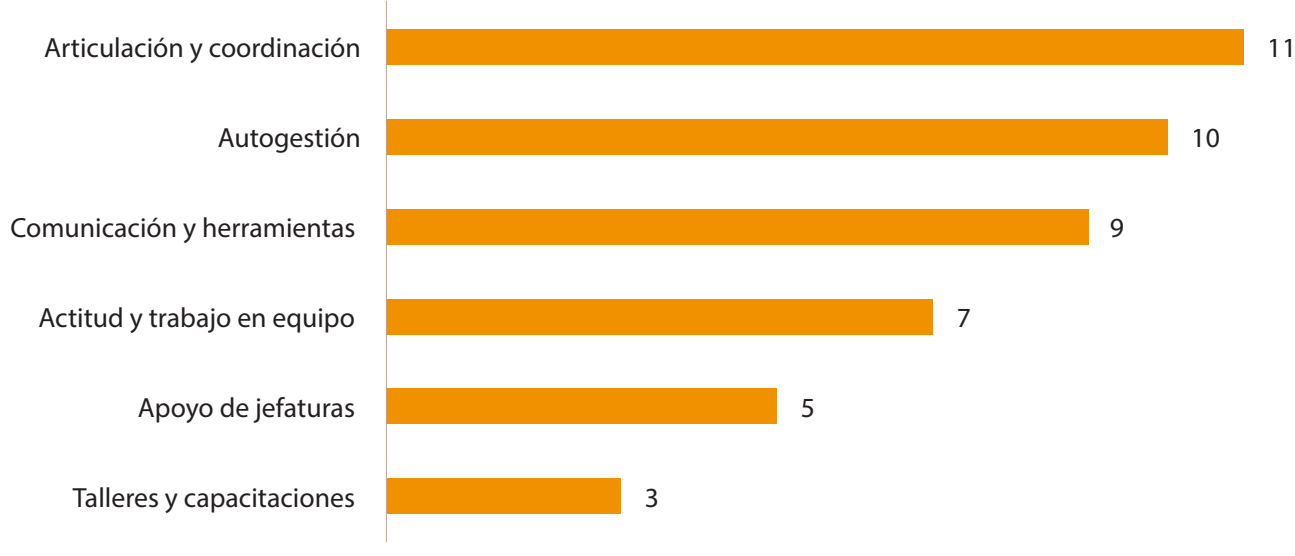

Nota: Elaboración propia con base en el instrumento (*Representado en números reales). 
http://doi.org/10.15359/ree.25-3.12

http://www.una.ac.cr/educare

educare@una.ac.cr

En torno a las estrategias empleadas por las coordinaciones para la convocatoria y flujo de información, quienes participaron destacaron la realización y divulgación de cronogramas mensuales y anuales. Para el manejo de la información destacan la comunicación con jefaturas y entre los equipos de trabajo (Tabla 2).

Tabla 2: Estrategias empleadas por las coordinaciones para la convocatoria y flujo de información

\section{Estrategias para el manejo a convocatorias Estrategias para el manejo de información}

- Programación y agenda mensual.

- Autogestión y contextualización pertinente a la red local con un mínimo de convocatorias bimensuales.

- Apoyo de jefaturas, comunicación constante y tiempo de respuesta oportuna.

- Solicitud y manejo desde las direcciones regionales de cada institución.

- Diversos medios de comunicación en caso de reuniones locales.

- Cronograma de reuniones anual.

- Coordinaciones informan a las jefaturas.

- Comunicados vía instancias superiores.
- Información del nivel local se traslada a los demás niveles.

- Revisión de protocolos.

- Coordinaciones divulgan la información.

- Buscar el respaldo de las jefaturas.

- Divulgación y socialización de objetivos del equipo.

- Por medio de oficios a las instituciones.

- Involucrando al personal en general, y personal asesor, supervisor y directivo de las instituciones.

- Comunicar cronograma anual a jefaturas.

- Asignación de tareas en reuniones y a no presentes se les comunican.

- Realización de actas o informes de reuniones y actividades en los que se registra asistencia y evidencias, se evalúan logros y necesidades, son entregados a jefaturas.

- Sensibilización a personal director regional mediante comunicación activa.

- Divulgación de lineamientos desde oficinas locales y regionales.

Nota: Elaboración propia con base en el instrumento.

En cuanto a la gestión, planeamiento y procesos autoevaluativos desde la coordinación, se aprecia la variedad de herramientas y estrategias que se reportan como utilizadas por las RISA. Los protocolos de atención destacaron como herramientas para los procesos de gestión, y las bases de datos representaron insumos necesarios para asegurar los procesos de seguimiento de la atención del desarrollo. Las reuniones, talleres y capacitaciones también fueron identificados como actividades de gestión relevantes (Tabla 3).

Además, en la Tabla 3 se reporta que las herramientas de autoevaluación son parte del esfuerzo de las redes por consolidar y mejorar su trabajo. Resalta entre las estrategias el análisis de logros según el plan de trabajo, lo que implica una relación directa entre los procesos de planificación, gestión y autoevaluación. 
http://doi.org/10.15359/ree.25-3.12

Tabla 3: Gestión, planificación y procesos de autoevaluación desde la coordinación

\begin{tabular}{|c|c|c|}
\hline Herramientas de gestión & Estrategias de planificación & Herramientas de autoevaluación \\
\hline - Plan y cronograma de anual. & - Sensibilización al personal & - Informe de logros. \\
\hline \multirow{16}{*}{$\begin{array}{l}\text { - Libro de actas, bitácoras de } \\
\text { reuniones e informes de } \\
\text { reuniones y actividades. } \\
\text { - Base de datos. } \\
\text { - Protocolos. } \\
\text { - Comunicación y trabajo en equipo. } \\
\text { - Socialización de las reuniones de } \\
\text { coordinadores. } \\
\text { - Capacitaciones. } \\
\text { - Lista de control de referencias y } \\
\text { boletas. }\end{array}$} & \multirow{3}{*}{$\begin{array}{l}\text { - Definir las posibilidades de acción de } \\
\text { cada institución } \\
\text { - Promover la disposición al trabajo en } \\
\text { equipo y la comunicación activa }\end{array}$} & - Listas de asistencia. \\
\hline & & - Informe de actividades. \\
\hline & & $\begin{array}{l}\text { - Listas de referencias contra } \\
\text { citas asianadas. }\end{array}$ \\
\hline & - Plan anual & - Realización de FODA. \\
\hline & - Cronograma de reuniones y actividades & \multirow{2}{*}{$\begin{array}{l}\text { - Según evolución de casos se } \\
\text { evalúa la atención de los centros. }\end{array}$} \\
\hline & - Protocolos & \\
\hline & - Reuniones mensuales & \multirow{2}{*}{$\begin{array}{l}\text { - Análisis de logros según el } \\
\text { plan de trabajo. }\end{array}$} \\
\hline & - Informes de reuniones y actividades & \\
\hline & - Convocatorias formales & \multirow{8}{*}{$\begin{array}{l}\text { - Conversatorios: pertinencia } \\
\text { de acciones y efectividad de } \\
\text { protocolos }\end{array}$} \\
\hline & $\begin{array}{l}\text { - Incurrir legalmente en caso de que } \\
\text { amerite la defensa de derechos de los } \\
\text { niños y las niñas }\end{array}$ & \\
\hline & - Inducciones-Capacitaciones-Talleres & \\
\hline & - Conversatorios & \\
\hline & - Revisión y control de referencias y citas & \\
\hline & $\begin{array}{l}\text { - Vinculación de proyectos de promoción } \\
\text { entre instituciones }\end{array}$ & \\
\hline & $\begin{array}{l}\text { - Divulgación de información de } \\
\text { reuniones de coordinadores }\end{array}$ & \\
\hline & $\begin{array}{l}\text { - Uso de diversas herramientas } \\
\text { tecnológicas de comunicación }\end{array}$ & \\
\hline
\end{tabular}

Nota: Elaboración propia con base en el instrumento.

La Tabla 4 presenta la perspectiva de las personas participantes en torno a las fortalezas y debilidades de la red. Destaca la actitud positiva y de liderazgo de sus miembros como la mayor fortaleza. Le sigue la actitud de compromiso y responsabilidad por parte del equipo, así como el trabajo articulado. En torno a las debilidades del proceso, desde la perspectiva de las personas coordinadoras participantes, se destaca el recargo de funciones, así como las agendas comprometidas como la mayor debilidad. Igualmente, se reconoce la falta de compromiso de integrantes y personal de las RISA. 
http://doi.org/10.15359/ree.25-3.12

http://www.una.ac.cr/educare

educare@una.ac.cr

Tabla 4: Fortalezas y debilidades del proceso de acuerdo con las coordinaciones

\begin{tabular}{|c|c|c|c|}
\hline Fortalezas & $\mathbf{N}$ & Debilidades & $\mathbf{N}$ \\
\hline $\begin{array}{l}\text { Miembros y funcionariado (interés-motivación- } \\
\text { disposición-liderazgo-empatía) }\end{array}$ & 16 & Recargo de funciones y agendas comprometidas & 10 \\
\hline $\begin{array}{l}\text { Equipos de trabajo (Consolidados-Comprometidos- } \\
\text { Responsables) }\end{array}$ & 13 & Poco compromiso de integrantes y personal & 10 \\
\hline Trabajo articulado (multidisciplinario-interinstitucional) & 12 & Falta de tiempo para el trabajo en red & 6 \\
\hline Comunicación constante & 7 & Necesidad de profesionales en los equipos de trabajo & 5 \\
\hline Apoyo de las jefaturas & 6 & Implementación y mejora de protocolos & 5 \\
\hline Experiencia y formación del personal para el trabajo en red & 6 & Problemas para obtener permisos & 4 \\
\hline $\begin{array}{l}\text { Autogestión administrativa (herramientas tecnológicas- } \\
\text { protocolos-creación de documentos e instrumentos) }\end{array}$ & 4 & Dificultad con transporte y viáticos & 3 \\
\hline Detección, atención y seguimiento de casos & 4 & Ausentismo en convocatorias & 3 \\
\hline Capacitaciones al personal y a las familias & 3 & Falta de creación de instrumentos & 2 \\
\hline Funciones definidas y cumplimiento de protocolos & 3 & Distancias que afectan comunicación y atención oportuna & 2 \\
\hline $\begin{array}{l}\text { Disposición de recursos, espacios físicos y } \\
\text { ambientes propicios }\end{array}$ & 3 & Falta de recursos y espacio físico & 2 \\
\hline Identificar necesidades y priorizarlas & 2 & Poca experiencia previa en trabajo en red & 1 \\
\hline
\end{tabular}

Nota: Elaboración propia con base en el instrumento.

La capacitación y el crecimiento profesional destaca en la Tabla 5 como una de las oportunidades del proceso, al lado del trabajo coordinado entre instituciones y el apoyo de jefaturas. Por su parte, como amenazas del proceso destacan el ausentismo y la falta de compromiso, la ausencia de las condiciones necesarias para el trabajo en red, así como la limitación de recursos y presupuesto. 
http://doi.org/10.15359/ree.25-3.12

Tabla 5: Oportunidades y amenazas del proceso de acuerdo con las coordinaciones

\begin{tabular}{|c|c|c|c|}
\hline Oportunidades & $\mathbf{N}$ & Amenazas & $\mathbf{N}$ \\
\hline Capacitación y crecimiento profesional & 10 & Ausentismo y falta de compromiso & 9 \\
\hline Trabajo coordinado entre instituciones & 9 & $\begin{array}{l}\text { Coordinación y condiciones efectivas para el } \\
\text { trabajo en red }\end{array}$ & 8 \\
\hline Apoyo de jefaturas, directores y direcciones regionales & 8 & Falta de recursos y presupuesto & 7 \\
\hline Atención eficaz y oportuna al valorar, detectar y derivar & 6 & Inestabilidad del personal o falta de este mismo & 6 \\
\hline Atención intersectorial de casos que lo requieren & 6 & Falta de apertura de códigos y de servicios & 5 \\
\hline Sensibilización por la atención de la primera infancia & 5 & Incumplimiento de lineamientos y protocolos & 3 \\
\hline Colaboración y alianzas en desarrollo de proyectos & 5 & Dificultad de acceso a algunas zonas & 3 \\
\hline Seguimiento de procesos de atención & 3 & $\begin{array}{l}\text { Incorporación de más instituciones } \\
\text { competentes a la atención infantil }\end{array}$ & 1 \\
\hline Acceso y control de información & 3 & & \\
\hline Abarcar el territorio nacional & 3 & & \\
\hline Optimización de recursos & 2 & & \\
\hline Disposición política y aval del decreto & 2 & & \\
\hline Apertura de servicios en CCSS y MEP & 1 & & \\
\hline $\begin{array}{l}\text { Obtener un panorama regional del desarrollo de la } \\
\text { población infantil }\end{array}$ & 1 & & \\
\hline
\end{tabular}

Nota: Elaboración propia con base en el instrumento.

\section{Discusión y conclusiones}

Se discuten a continuación los resultados en torno a los tres ejes de preguntas planteadas a quienes participaron, a saber: el uso de instrumentos técnicos o de normalización para el trabajo en red; las características de la gestión de los equipos, y las fortalezas/debilidades, oportunidades/ amenazas identificadas por quienes participaron. Esta información permite caracterizar el modelo de gestión de la experiencia en estudio desde la perspectiva de sus actores. 
http://doi.org/10.15359/ree.25-3.12

http://www.una.ac.cr/educare

educare@una.ac.cr

Caracterización de los documentos técnicos que utilizan las redes. Se destacó el uso de la «Boleta de Referencia y Contrarreferencia». Este resultado evidencia la función misma de las redes. Ellas están creadas para "promover, mantener y/o recuperar el desarrollo integral de las personas de 0 a 12 años" (CCSS, 2012, p. 45). La boleta es el instrumento básico para la labor de referencia y contrarreferencia que intercambian las personas profesionales de las distintas disciplinas. Por otra parte, la implementación de estrategias de detección temprana es también parte significativa de la labor de las redes para el ejercicio de referencia y contrarreferencia, lo que puede explicar el valor que las redes confieren al documento de Lineamientos para la Valoración del Desarrollo y al Expediente Educativo del MEP. Precisamente, los instrumentos que se califican como los de mayor utilización corresponden a algunas de las acciones propias de las RISA, a saber, detección temprana, manejo de cada caso y derivación según criterios de referencia (CCSS et al., 2016).

Gestión de los equipos. En relación con los mecanismos, actividades y estrategias implementadas por miembros de la red para mejorar la atención de las personas participantes, los procesos de autogestión fueron identificados como los mecanismos o estrategias más utilizados. Este resultado evidencia una dinámica bastante autónoma de las redes. La naturaleza misma del sistema requiere la autogestión de las redes dentro de un marco de objetivos comunes, en concordancia con su génesis y considerando que "la atención integral, no es una tarea que pueda asumir una sola organización, sector o contexto, sino que supone la participación de todos desde la definición de objetivos e intervenciones específicas" (CCSS et al., 2012, p. 36).

El fenómeno descrito está ligado con los datos sobre las estrategias utilizadas por las redes para contrarrestar la organización institucional lineal, centralista y desarticulada que afecta la gestión en red. De nuevo la autogestión, así como la articulación y coordinación aparecen como las estrategias más utilizadas. Como apuntaban Urban et al. (2012), un elemento clave de un modelo adecuado es contar con soporte a quienes lo integren, de tal forma que se promuevan prácticas responsables y receptivas capaces de dar atención a las necesidades de la niñez, de las familias y sus comunidades. En este sentido, un sistema de atención bien articulado, como también indican Kaga et al. (2010), representa un vehículo para la profesionalización y el desarrollo de competencias de quienes trabajan con la niñez.

En torno a las estrategias empleadas por las coordinaciones para la convocatoria y flujo de información, quienes participaron destacaron el uso de cronogramas mensuales y anuales y la comunicación con jefaturas y entre los equipos de trabajo para el manejo de la información. Las convocatorias de miembros de las redes implican un proceso de planificación, tanto de los niveles locales como de los niveles regionales. Pero algo que resulta interesante de los datos arrojados es la dirección del flujo de la información. En un modelo centralista y jerárquico, la comunicación siempre se da de arriba hacia abajo, es decir, desde los puestos de mando hacia los puestos más operativos. Sin embargo, la información recabada arroja un movimiento fuerte 
http://doi.org/10.15359/ree.25-3.12

de información desde los niveles locales hacia los niveles de jefaturas. Por ejemplo, uno de los informantes expresa que una de las estrategias utilizadas para el manejo de la información es "comunicar cronograma anual a jefaturas". Es decir, la red local no espera que los procesos sean planteados por los niveles superiores, sino que, como apunta el CCSS et al. (2012), las redes deben dar respuesta a las necesidades del entorno, de la familia y de la comunidad. Este fenómeno da cuenta de la dinámica del trabajo articulado intersectorial, donde el modelo de gestión es mucho más permeable y flexible que los modelos tradicionales. Los datos recabados muestran el apoyo de las jefaturas para alcanzar los objetivos propuestos. Al respecto, uno de los informantes señala como estrategia para el manejo de información se debe "buscar el respaldo de las jefaturas". De nuevo, las habilidades de liderazgo y comunicación al interior de las redes se muestran como un componente indispensable en los procesos de gestión que deben fluir en ambas direcciones. En concordancia con Dabas y Perrone (1999), se requiere de capacidad resolutiva, lo que significa que no puede esperar de permisos para ser eficaces y eficientes.

En relación con la gestión, planificación y procesos de autoevaluación desde la coordinación, los protocolos de atención se destacaron como recursos que clarifican la competencia y labor de cada institución y miembro de los equipos. Las bases de datos también representaron insumos necesarios para asegurar los procesos de seguimiento de la atención del desarrollo. Las reuniones, los talleres y las capacitaciones fueron identificados como estrategias que aportan directamente al crecimiento del recurso humano que participa en las redes. Este hecho cobra especial relevancia en función de los relativos niveles de autonomía que ejercen las personas participantes de las RISA locales. De manera que personas funcionarias con alto criterio técnico y profesional podrían dar respuestas pertinentes a las necesidades de los niños, las niñas y sus familias, más aún en un entorno de equipo. En relación con los procesos de capacitación, la promoción del liderazgo en los equipos parte de la premisa de que la formación continua representa una herramienta fundamental para homogeneizar el manejo conceptual entre los equipos y disciplinas en torno a la gestión técnica y administrativa. Estos resultados contrastan con el modelo del Sistema Intersectorial de Protección Social de Chile, que reporta una articulación nacional vertical. Como Cunill-Grau (2014) reconoció, "en vez de un modelo descentralizado opera una dinámica de arriba-abajo, en la que se busca una lógica de integración centralmente dirigida" (p. 29). Si bien los resultados del proyecto INTESYS en el que participaron varios países europeos revelaron que no existe una única vía para consolidar una propuesta de atención intersectorial, la descentralización y la verticalidad resaltan como características de un modelo eficiente de atención a la niñez.

Fortalezas, debilidades, oportunidades y amenazas del proceso. Como fortaleza destacó la actitud positiva y de liderazgo de los miembros de los equipos. Le sigue la actitud de compromiso y responsabilidad por parte del equipo, así como el trabajo articulado. En torno a 
http://doi.org/10.15359/ree.25-3.12

http://www.una.ac.cr/educare

educare@una.ac.cr

las debilidades del proceso, se destacó el recargo de funciones y las agendas comprometidas. Igualmente, se reconoce la falta de compromiso de integrantes y personal de las RISA. Este resultado contrasta con el personal comprometido y motivado que es también la principal fortaleza del proceso. De manera que el recurso humano aparece como la principal fortaleza y también como la principal debilidad del proceso. Se puede desprender, sin embargo, que el recargo de tareas para quienes asumen las funciones de articulación tiene un peso importante en la valoración de las personas participantes. Otras dificultades de gestión se suman a las debilidades reconocidas, como las dificultades para obtener permisos, transportes o viáticos, sumado a la falta de recursos y espacio físico. De esto se desprende la necesidad de políticas públicas que aseguren una mejora en la carga laboral, y que al reconocer la importancia de participar en estos procesos para quienes atienden a la niñez. De la misma manera, los esfuerzos de la comunidad internacional por fomentar el cuidado y educación de la niñez han de tomar en cuenta el bienestar y capacitación de quienes la atienden.

Por otra parte, los resultados arrojaron que la capacitación y el crecimiento profesional, al igual que el trabajo coordinado entre instituciones y el apoyo de jefaturas fueron las mayores oportunidades que las participantes encontraron en su participación en las redes. De acuerdo con el CCSS et al. (2012), la atención integral de la niñez requiere "de una comprensión sistémica y compleja del quehacer institucional a favor del bienestar poblacional" (p. 36). El sistema representa un modelo de gestión que ofrece oportunidades de crecimiento personal y colectivo, como reconocen los sujetos informantes. Los diferentes niveles de atención tienen la oportunidad para desarrollar estilos de liderazgo que faciliten los procesos y el flujo de la información en ambos sentidos. La disposición de las personas profesionales de salud y educación participantes en las redes tiene una oportunidad para desarrollarse en torno a la atención integral de la niñez. Así lo señalan quienes destacan la sensibilización por la atención a la primera infancia, la atención y seguimiento de casos o el desarrollo de un panorama más amplio en torno al desarrollo de la población infantil.

Como amenazas del proceso destacaron el ausentismo y la falta de compromiso, la falta de las condiciones necesarias para el trabajo en red, así como la falta de recursos y presupuesto. De acuerdo con el CCSS et al. (2012), "[la] atención integral refiere a la capacidad material, humana, financiera y conceptual de las [instituciones] para definir las estrategias de abordaje dirigidas a la niñez" (p. 35). Es decir, las estructuras institucionales tradicionales y los modelos de liderazgo rígidos y verticales pueden amenazar el funcionamiento de las redes y del sistema. Precisamente, la posibilidad de compartir recursos, visiones y conocimientos constituye un reto para un tipo de gestión que se resiste a confiar en un sistema competente, como lo llaman Urban et al. (2012), el cual solo puede funcionar en un contexto de relaciones de carácter recíproco entre público usuario, equipos de trabajo e instituciones dentro de un contexto social y político. 


\section{Conclusiones}

A partir del análisis de estos resultados, es posible establecer algunas caracterizaciones del modelo de gestión para la atención de la primera infancia en Costa Rica, desde la perspectiva de las personas coordinadoras participantes. El modelo de gestión funciona en red, en la cual participan profesionales de salud y educación de tres niveles articulados interinstitucionalmente. En la esfera local se utilizan documentos técnicos que permiten una labor sustantiva: la detección temprana de alteraciones del desarrollo, así como la referencia y contrarreferencia que intercambian las personas profesionales de las distintas disciplinas.

La gestión de las redes evidencia una dinámica bastante autónoma. La autogestión, así como la articulación y coordinación son estrategias que caracterizan el funcionamiento de las redes. Se utilizan múltiples documentos y estrategias para la planificación y la coordinación que denotan un flujo de la información en doble vía (de nivel de mando inferior al superior y viceversa). En este sentido, es posible visualizar el trabajo en red del SAIID desde un modelo de gestión que contrasta con los modelos tradicionales centralistas o verticales y que muestra ser más permeable y flexible. Una de las características más sobresalientes del trabajo en red es su necesidad de liderazgos en los diferentes niveles de organización del sistema. Estos liderazgos requieren de capacidad resolutiva que aseguren la eficacia y eficiencia de la atención. Es así como, pese a la relativa autonomía que requieren las redes para ser eficaces y eficientes en su labor de atención a la niñez, se reconoce la necesidad del apoyo de las jefaturas para alcanzar los objetivos propuestos. El sector educativo y las personas profesionales en salud, al trabajar de forma conjunta y coordinada, mejoran las intervenciones en sus comunidades (Burgess et al., 2016).

Las fortalezas, debilidades, oportunidades y amenazas de las redes apuntan a la importancia del factor humano en el funcionamiento de las redes. En este sentido, se reconoce como necesario el énfasis en las capacitaciones y la formación de quienes conforman el sistema, precisamente como forma de promoción del liderazgo. La promoción del liderazgo en los equipos parte de la premisa de que la formación continua representa una herramienta fundamental para homogeneizar el manejo conceptual entre los equipos y disciplinas en torno a la gestión técnica y administrativa. Esta estrategia asegura un modelo sostenible que da respuesta a las necesidades de atención de la población infantil. Los modelos de gestión estatal de la atención de la niñez sufren así transformaciones que requieren de una disposición al cambio tanto de las jefaturas como de las personas profesionales que conforman las redes locales de atención directa al usuario. La construcción de un modelo intersectorial de redes de atención desde el Estado, que consolide la propuesta, y que sirva como base es el camino que ha mostrado éxito en países desarrollados (Loewenson y World Health Organization, 2013). Un modelo de gestión que incluye la formación continua de quienes son parte del sistema, resulta un modelo sostenible que da respuesta a los llamados de la comunidad nacional e internacional por el cuidado y la educación de la infancia. 
http://doi.org/10.15359/ree.25-3.12

http://www.una.ac.cr/educare

educare@una.ac.cr

Este estudio debe ampliarse en cuanto a los cambios en el modelo de atención de la niñez que ha experimentado Costa Rica en años recientes. Se necesita establecer una evaluación periódica y sistemática del funcionamiento de las redes. Así mismo, otras investigaciones deben realizar evaluaciones del impacto a nivel de indicadores de salud y educativos que ha tenido la conformación de redes intersectoriales. Finalmente, desde el ámbito docente, se debe ahondar sobre el alcance que el trabajo intersectorial tiene en esta esfera profesional, y el papel de la formación en temas como liderazgo y habilidades blandas, competencias fundamentales para enfrentar los nuevos desafíos educativos.

\section{Declaración de Material complementario}

Este artículo tiene disponible, como material complementario:

-La versión preprint del artículo en https://doi.org/10.5281/zenodo.4907109

\section{Referencias}

Alvira Martín, F. (2011). La encuesta: Una perspectiva general metodológica (2. ${ }^{a}$ ed.). Centro de Investigaciones Sociológicas.

Anaf, J., Baum, F., Freeman, T., Labonte, R., Javanparast, S., Jolley, G., Lawless, A. y Bentley, M. (2014). Factors shaping intersectoral action in primary health care services. Australian and New Zealand Journal of Public Health, 38(6), 553-559. https://doi.org/10.1111/17536405.12284

Bilodeau, A., Laurin, I., Giguère, N.y Potvin, L. (2018). Understanding the challenges of intersectoral action in public health through a case study of early childhood programmes and services. Critical Public Health, 28(2), 225-236. https://doi.org/10.1080/09581596.2017.1343934

Burgess, T., Braunack-Mayer, A., Tooher, R., Collins, J., O'Keefe, M., Skinner, R., Watson, M., Ashmeade, H., Proeve, C. y Marshall, H. (2016). Optimizing intersectoral collaboration between health and education: the Health Bridges study. Journal of Public Health, 38(4), e430-c437. https://doi.org/10.1093/pubmed/fdv190

Caja Costarricense de Seguro Social, Ministerio de Educación Pública, Ministerio de Salud, Dirección Nacional CEN-CINAI, Universidad Estatal a Distancia, Universidad de Costa Rica, Universidad Nacional. (2016). Protocolo intersectorial de atención al desarrollo de niñas y niños preescolares y escolares. Imprenta CCSS. 
http://doi.org/10.15359/ree.25-3.12

http://www.una.ac.cr/educare educare@una.ac.cr

Caja Costarricense de Seguro Social, Ministerio de Educación Pública, Ministerio de Salud, Universidad Estatal a Distancia, Universidad de Costa Rica, Universidad Nacional. (2012). Atención integral del desarrollo de niñas y niños en Costa Rica: Fortalecimiento intersectorial. Imprenta CCSS. https://docplayer.es/3982407-Atencion-integral-del-desarrollo-de-ninasy-ninos-en-costa-rica-fortalecimiento-fortalecimiento-intersectorial.html

Cea D’Ancona, M. Á. (1998). Metodología cuantitativa: Estrategias y técnicas en investigación social. Síntesis.

Cerdas González, A. I. (2016). Lecciones aprendidas en el diseño e implementación del Sistema de Atención Integral e Intersectorial del Desarrollo del Niño y la Niña 2005-2016. Experiencia de Costa Rica. https://docplayer.es/90721828-Lecciones-aprendidas-en-el-diseno-eimplementacion-del-sistema-de-atencion-integral-e-intersectorial-del-desarrollo-delnino-y-la-nina.html

Creswell, J. W. (2008). Educational research: Planning, conducting, and evaluating quantitative and qualitative research (3. ${ }^{a}$ ed.). Pearson.

Cunill-Grau, N. (2014). La intersectorialidad en las nuevas políticas sociales: Un acercamiento analítico-conceptual. Gestión y Política Pública, 23(1), 5-46. http://www.scielo.org.mx/ scielo.php?script=sci arttext\&pid=S1405-10792014000100001\&lng=es\&tlng=es

Dabas, E. y Perrone, N. (1999). Redes en salud. En E. Dabas (Comp.), Viviendo en redes. Experiencias y estrategias para fortalecer la trama social. Editorial CICCUS.

Fondo de las Naciones Unidas para la Infancia y Sistema Nacional para el Desarrollo Integral de la Familia. (2001). Observaciones generales del Comité de los Derechos del Niño. Autor. https://www.unicef.org/UNICEF-ObservacionesGeneralesDelComiteDeLosDerechosDelN ino-WEB.pdf

Hernández Sampieri, R., Fernández Collado, C. y Baptista Lucia, P. (2014). Metodología de la investigación (6. ${ }^{\mathrm{a}}$ ed.). McGraw-Hilla.

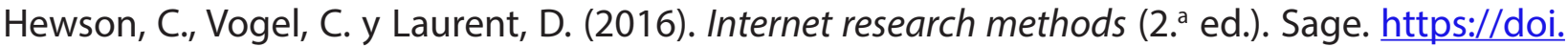
org/10.4135/9781473920804

Kaga, Y., Bennett, J. y Moss, P. (2010). Caring and learning together. A cross-national study of integration of early childhood care and education within education. UNESCO. https:// unesdoc.unesco.org/ark:/48223/pf0000187818

Kagan, S. L. y Kauerz, K. (2012). Early childhood systems: Looking deep, wide, and far. En S. L. Kagan, y K. Kauerz (Eds.), Early childhood systems: Transforming early learning (pp. 3-17). Teachers College Press. 
http://doi.org/10.15359/ree.25-3.12

http://www.una.ac.cr/educare

educare@una.ac.cr

Loewenson, R.y World Health Organization. (2013). Evaluating intersectoral processes for action on the social determinants of health: Learning from key informants. World Health Orgnization. http://apps.who.int/iris/handle/10665/84373

Pucher, K. K., Candel, M. J.J. M., Krumeich, A., Boot, N. M. W. M.y de Vries, N. K. (2015). Effectiveness of a systematic approach to promote intersectoral collaboration in comprehensive school health promotion-a multiple-case study using quantitative and qualitative data. BMC Public Health, 15(1), 1-14. https://doi.org/10.1186/s12889-015-1911-2

Rentzou, K., lonescu, M., Zimmerli, P. y Boca, C. (2019). La coordinación intersectorial en los sistemas para la primera infancia: El enfoque Primokis. Early Childhood Matters. https:// earlychildhoodmatters.online/2019/inter-sectoral-coordination-in-early-childhoodsystems-the-primokiz-approach/?ecm2019

Rovai, A. P., Baker, J. D. y Ponton, M. K. (2013). Social science research design and statistics: A practitioner's guide to research methods and SPSS analysis. Watertree Press.

Secretaría de Educación Pública. (2010). Modelo de gestión educativa estratégica (2a ed.). Autor. http://www.ses/p.gob.mx/pdf/taller2011-2012/uno/DOCUMENTOS/9915-Modelo\%20 de\%20Gestion\%20EducativaFINAL.pdf

Tooher, R., Collins, J., Braunack-Mayer, A., Burgess, T., Skinner, S. R., O'Keefe, M., Watson, M. y Marshall, H. S. (2017). Intersectoral collaboration to implement school-based health programmes: Australian perspectives. Health Promotion International, 32(2), 312-321. https://doi.org/10.1093/heapro/dav120

Urban, M., Vandenbroeck, M., van Laere, K., Lazzari, A. y Peeters, J. (2012). Towards competent systems in early childhood education and care. Implications for policy and practice. European Journal of Education, 47(4), 508-526. https://doi.org/10.1111/ejed.12010

Zero to Three. (3 de octubre de 2020). Early childhood systems. https://www.zerotothree.org/ espanol/early-childhood-systems 